\title{
§ 6 Völkergewohnheitsrechtliche Geltung
}

Die bereits im ersten Kapitel nachgewiesenen Kodifizierungen des Rechts auf Nahrung in staatlichen Verfassungen und völkerrechtlichen Verträgen (§ $1 \mathrm{~A})$ und die im fünften Kapitel dargelegte Rechtsprechung zu den drei Zugangsrechten (§ 5) sprechen für eine gewohnheitsrechtliche Geltung des „right to food“, so dass alle Völkerrechtssubjekte, einschließlich der Staaten, die nicht Vertragsparteien des UN-Sozialpakts geworden sind, und Internationale Organisationen an dieses gebunden wären.

Die jüngere Literatur nimmt eine solche auf Gewohnheit beruhende Geltung des Rechts auf Nahrung an. ${ }^{1}$ Dieses wird auch als allgemeiner Rechtsgrundsatz bezeichnet. ${ }^{2}$ Letztere Einstufung erscheint angesichts des dynamischen Charakters des ,right to food“ jedoch fragwürdig. Statt dieses als starres Rechtsprinzip zu beschreiben, sollen in diesem Kapitel die unterschiedlichen Facetten herausgearbeitet werden, die sich in der völkerrechtlichen Praxis als Mindestanforderungen etabliert haben. Wie zu zeigen sein wird, besteht die Herausforderung weniger in der Nachweisführung der gewohnheitsrechtlichen Geltung (A) als in der Bestimmung des genauen Norminhalts (B).

\section{A. Nachweis}

Die Entstehung einer gewohnheitsrechtlichen Norm setzt allgemein eine von einer Rechtsüberzeugung (opinio iuris) getragene Staatenpraxis (consuetudo) voraus. ${ }^{3}$ In diesem Sinne beschreibt Art. 38 Abs. 1 lit. b) des

1 Siehe Kearns, A. P. (1998): "The Right to Food Exists Via Customary International Law". In: Suffolk Transnational Law Review 22, S. 223; Narula, S. (2006): "The Right to Food: Holding Global Actors Accountable Under International Law". In: Columbia Journal of Transnational Law, Vol. 44, S.691f.; Niada, L. (2006): "Hunger and International Law: The Far-Reaching Scope of the Human Right to Food". In: 22 Connecticut Journal of International Law 131, S. 131 (173ff.).

2 Eckardt, F. \& Hyla, A. (2010): „Menschenrechte, Welthandel und das Recht auf Nahrung“. In: Zeitschrift für Menschenrechte 4, Nr. 2, S. 73 (85).

3 Statt aller Verdross, A. \& Simma, B. (1984): Universelles Völkerrecht, Theorie und Praxis, 3. Auflage, $\S 551$. 
IGH-Statuts das internationale Gewohnheitsrecht als ungeschriebene Rechtsquelle, die Ausdruck einer allgemeinen als Recht anerkannten Übung ist.

\section{Staatenpraxis}

Die geschätzte Zahl von 795 Millionen weltweit hungernden Menschen spricht in deutlicher Weise gegen eine ausreichende Staatenpraxis, die das Recht auf Nahrung für alle garantieren könnte. Die weiterhin hohen Hungerzahlen können jedoch nicht als Beleg angeführt werden, um die weltweiten Bemühungen, einen Zustand der Nahrungssicherheit zu erreichen, generell in Abrede zu stellen. So haben alle Staaten, die die Sonderberichterstatter für das Recht auf Nahrung Ziegler und de Schutter besuchten, Strategien zur Nahrungssicherheit erarbeitet und implementiert. ${ }^{4}$ Die Vereinigten Staaten von Amerika, die sich lange gegen die Verbindlichkeit des Rechts auf Nahrung gewandt haben, verfolgen seit jeher sowohl auf nationaler ${ }^{5}$ als auf internationaler ${ }^{6}$ Ebene eine systematische Nahrungssicherheitspolitik. $^{7}$

Als wichtige Institution auf internationaler Ebene ist neben der Ernährung- und Landwirtschaftsorganisation der Vereinten Nationen (FAO) ${ }^{8}$ das Welternährungsprogramm (World Food Programme) zu nennen, das seit 1961 die größte humanitäre Organisation der Vereinten Nationen darstellt. Jährlich werden Nahrungsmittelhilfen für durchschnittlich 90 Mio. Menschen in mehr als 70 verschiedenen Ländern bereitgestellt. ${ }^{9}$ Bedeutsam ist ebenso der Internationale Fonds für landwirtschaftliche Entwick-

4 Beide haben mit ihren Teams jeweils zwölf Ländermissionen unternommen, alle Berichte sind abrufbar unter: < http://www.ohchr.org/EN/Issues/Food/Pages/Visits.a $\mathrm{spx}>$.

5 Das Supplemental Nutrition Assistance Program stellt Nahrungshilfen für Millionen gering verdienende Personen und Haushalte in den USA bereit, Angaben auf Homepage des US-Landwirtschaftsministeriums: <www.fns.usda.gov/snap/supple mental-nutrition-assistance-program-snap>.

6 Die US-Entwicklungsbehörde setzt sich weltweit für Nahrungssicherheit ein und koordiniert die internationale Nahrungsmittelhilfe, siehe $<$ http://www.usaid.gov/wh at-we-do/agriculture-and-food-security>.

7 Vgl. Nachweise bei Narula 2006 (Fn. 1), S. 83.

8 Oben $\S 3$ A II (S. 84ff.).

9 Angaben auf der Homepage des World Food Programes: <www.wfp.org>. 
lung (International Fund for Agricultural Development), der seit 1977 Kredite zur Stärkung der Agrarproduktion vergibt. ${ }^{10}$

\section{Rechtsüberzeugung}

Michael Dennis und David Stewart führen die Millenniums-Erklärung der Vereinten Nationen als Nachweis für die fehlende Überzeugung der Staatengemeinschaft von der juridischen Verbindlichkeit des Rechts auf Nahrung an. ${ }^{11}$ Auch wenn das Ziel der Halbierung der Hungerzahlen bis 2015 offensichtlich ungenügend ist, um das Recht auf Nahrung für alle zu verwirklichen, verkehrt es jedoch den Sinn der Erklärung, diese als implizite Ablehnung einer menschenrechtlichen Verpflichtung zu interpretieren. Im Gegenteil bekräftigt die Jahrtausenderklärung ausdrücklich die bestehenden Verpflichtungen und Bekenntnisse. ${ }^{12}$ Die neuen UN-Ziele nachhaltiger Entwicklung enthalten zudem das eindeutige Gebot, den Hunger in der Welt bis 2030 vollständig zu beseitigen ${ }^{13}$ und verweisen explizit auf die Geltung der Menschenrechte. ${ }^{14}$

\section{Kodifizierungen}

Die im ersten Kapitel aufgezeigten expliziten Anerkennungen auf nationaler, regionaler und internationaler Ebene belegen die weltweite Überzeugung von der Gültigkeit des Rechts auf Nahrung. ${ }^{15}$ So hat die Interamerikanische Menschenrechtskommission im „Domingues“-Fall (2002) die völkergewohnheitsrechtliche Geltung des Verbots der Todesstrafe gegen Jugendliche unter 18 Jahren maßgeblich auf dessen völkerrechtliche Posi-

10 Die seit seiner Gründung eingesetzten Mittel des IFAD belaufen sich auf $13 \mathrm{Mrd}$. US-Dollar, die für 870 Projekte, insbesondere in Subsahara Afrika, eingesetzt wurden, Angaben auf der IFAD-Homepage: <www.ifad.org/governance/faq/index .htm> (Stand Mai 2014).

11 Dennis \& Stewart 2004 (oben § 4 Fn. 93), S. 496.

12 Vgl. UN Secretary-General 2001 (oben $\S 3$ Fn. 11), Rn. 7.

13 UNGA ( 33 Fn. 14), S. 15.

14 Ebd., Rn. 8.

15 Oben $\S 1$ A (S. 33ff.). 
tivierung in Art. 6 Abs. 5 IPbpR und zwei regionalen Menschenrechtsprotokollen gestützt. ${ }^{16}$

\section{Allgemeine Menschenrechtserklärung und UN-Resolutionen}

Schon die Allgemeine Menschenrechtserklärung von 1948 bekennt sich zum Recht auf Nahrung (Art. 25 I, erster Halbsatz), das vom Recht auf einen angemessenen Lebensstandard umfasst sein soll. Auch wenn dieser Erklärung keine formelle Rechtsverbindlichkeit zukommt, ist sie wie andere Resolutionen der UN-Generalversammlung als Hinweis für die internationale Akzeptanz einer Norm zu verstehen. So hat der Internationale Gerichtshof im Nicaragua-Fall (1986) zur Begründung der gewohnheitsrechtlichen Geltung des Gewaltverbots auf die Anerkennung desselben durch die „Friendly Relations“-Resolution von 1970 verwiesen. ${ }^{17}$

Seit 2001 verabschiedet die UN-Generalversammlung jedes Jahr im Dezember eine Resolution zum Recht auf Nahrung, die Art. 11 Abs. 1 und 2 IPwskR bekräftigen. ${ }^{18}$ Internationale Deklarationen, die sich im Rahmen der Welternährungsorganisation (FAO) zum Recht auf Nahrung bekennen, ${ }^{19}$ sprechen ebenfalls für einen überwältigenden Konsens der Staatengemeinschaft von der Gültigkeit des ,right to food“. ${ }^{20}$ Die UNMenschenrechtskommission ${ }^{21}$ und ihre Nachfolgeinstitution, der UN-

16 Inter-American Commission on Human Rights, Report $N^{\circ}$ 62/02, Case 12.285, Michael Domingues v. United States, Entsch. v. 22.10.2002, Rn. 55ff. und 84.

17 IGH, Case Concerning Military and Paramilitary Activities in and against Nicaragua [Nicaragua v. USA], ICJ Reports 1986, S. 14, Nr. 188.

18 UNGA, Resolution v. 19.12.2001, A/RES/56/155; Resolution v. 18.12.2002, A/RES/57/226; Resolution v. 22.12.2003, A/RES/58/186; Resolution v. 20.12.2004, A/RES/59/202; Resolution v. 16.12.2005, A/RES/60/165; Resolution v. 19.12.2006, A/RES/61/163; Resolution v. 18.12.2007, A/RES/62/164; Resolution v. 18.12.2008, A/RES/63/187; Resolution v. 18.12.2009, A/RES/64/159; Resolution v. 21.12.2010, A/RES/65/220; Resolution v. 19.12.2011, A/RES/ 66/158; Resolution v. 20.12.2012, A/RES/67/174; Resolution v. 18.12.2013, A/RES/68/177; Resolution v. 18.12.2014, A/RES/69/177.

19 Siehe oben $\S 2$ B I (S. 59ff.).

20 Vgl. Narula 2006 (Fn. 1), S. $76 f f$.

21 UNCHR, Resolution v. 17.04.2000, 2000/10, Rn. 2; Resolution v. 20.04.2001, 2001/25, Rn. 2; Resolution v. 22.04.2002, 2002/25, Rn. 2; Resolution v. 22.04.2003, 2003/25, Rn. 2. 
Menschenrechtsrat, ${ }^{22}$ haben ebenso zahlreiche Resolutionen verabschiedet, die sich auf Art. 11 Abs. 1 und 2 IPwskR beziehen.

\section{Ausnahme für Nicht-Vertragsstaaten des UN-Sozialpakts?}

$\mathrm{Zu}$ fragen bleibt, ob auch Staaten, die nicht Vertragspartei des UN-Sozialpakts geworden sind, gewohnheitsrechtlich an das Recht auf Nahrung gebunden sind. In Ost- und Südasien fehlt eine regionaler Menschenrechtsvertrag, der das „right to food“ verbürgen könnte. Dies spricht jedoch nicht gegen die universelle Anerkennung, weil sämtliche ost- und zentralasiatischen Staaten und der überwiegende Teil der süd- und südostasiatischen Staaten den UN-Sozialpakts ratifiziert haben. ${ }^{23}$ Zudem haben neun Mitgliedstaaten der Association of Southeast Asian Nations (AEAN) in einer gemeinsamen Menschenrechtserklärung vom November 2012 das Recht auf Nahrung bekräftigt. ${ }^{24}$

Als einzige Staaten, die weder den UN-Sozialpakt noch einen anderen das Recht auf Nahrung beinhaltenden Menschenrechtsvertrag ratifiziert haben, verbleiben allein zwei Länder: die Vereinigten Staaten von Amerika und der Inselstaat Palau. ${ }^{25}$ Die USA waren das einzige Land, das die Verbindlichkeit des Rechts auf Nahrung offiziell abgelehnt hat. ${ }^{26}$ Die Obama-Administration hat mittlerweile den Widerstand aufgegeben, so dass

22 HRC, Resolution v. 27.03.2008, A/HRC/RES/7/14, Resolution v. 26.03.2009, A/HRC/RES/10/12; Resolution v. 24.03.2010, A/HRC/RES/13/4; Resolution v. 25.03.2011, A/HRC/RES/16/27; Resolution v. 22.03.2012, A/HRC/RES/19/7; Resolution v. 27.03.2014, A/HRC/RES/25/14; Resolution v. 26.03.2015, $\mathrm{A} / \mathrm{HRC} / \mathrm{RES} / 28 / 10$.

23 In Südasien ist Bhutan als einziges Land nicht Vertragspartei des IPwskR; in Südasien sind es vier Staaten (Brunei, Malaysia, Myanmar und Singapur), vgl. U.N.T.C., Chapter IV, No. 3 (Stand Mai 2015).

24 ASEAN Human Rights Declaration, 19 November 2012, Rn. 28: "Every person has the right to an adequate standard of living for himself or herself and his or her family including: a. The right to adequate and affordable food, freedom from hunger and access to safe and nutritious food."

25 Iran, Somalia und der Sudan sind zwar nicht der Frauenrechtskonvention beigetreten, haben aber den IPwskR ratifiziert, vgl. U.N.T.C., Chapter IV, No. 3 (Stand Mai 2015).

26 Vgl. Nachweise bei Narula 2006 (Fn. 1), S. 83. 
nicht mehr entschieden werden muss, ob sich die USA als einziges Land auf die umstrittene Rechtsfigur des ,persistent objector" berufen könnte. ${ }^{27}$

Die US-Regierung hat sich erstmals im November 2009 zum Recht auf Nahrung durch vorbehaltslose Unterzeichnung der Abschlusserklärung des FAO-Welternährungsgipfels bekannt. ${ }^{28}$ Am 19. Dezember 2009 trugen die Vereinigten Staaten eine Resolution über das „right to food“ mit, die zum ersten Mal in der Geschichte der Vereinten Nationen im Konsens angenommen wurde. Darin wurde bekräftigt,

dass Hunger eine Schande ist und eine Verletzung der Menschenwürde darstellt und dass daher auf nationaler, regionaler und internationaler Ebene dringend Maßnahmen zu seiner Beseitigung ergriffen werden müssen;

das Recht jedes Menschen auf Zugang zu gesundheitlich unbedenklichen, ausreichenden und nährstoffreichen Nahrungsmitteln im Einklang mit dem Recht auf angemessene Ernährung und dem grundlegenden Recht eines jeden, vor Hunger geschützt zu sein, um seine körperlichen und geistigen Fähigkeiten in vollem Umfang entfalten und erhalten zu können. ${ }^{29}$

\section{B. Inhaltliche Reichweite}

$\mathrm{Zu}$ bestimmen bleibt der Verpflichtungsumfang der gewohnheitsrechtlichen Geltung. Nach Smita Narula hat zumindest das Recht, frei von Hunger zu leben (Art. 11 II IPwskR), einen völkergewohnheitsrechtlichen Status erlangt. ${ }^{30}$ Darunter versteht sie, im Unterschied zum Recht auf angemessene Ernährung (Art. 11 I IPwskR), einen absoluten Standard, der alle Staaten verpflichtet, die notwendigen Schritte zur Verwirklichung einzuleiten und international zu kooperieren:

27 Im Ergebnis ablehnend, ebd.

28 FAO, Declaration on Food Security 2009 (oben $\S 2$ Fn. 47), Rn. 2: "We must collectively accelerate steps (...) to set the world on a path to achieving the progressive realization of the right to adequate food in the context of national food security."

29 UNGA, Resolution v. 18.12.2009, A/RES/64/159, Nr. 1 und 2, Übersetzung nach Vereinte Nationen, Deutscher Übersetzungsdienst: <www.un.org/Depts/german/in dex.html>.

30 Narula 2006 (Fn. 1), S. 80 mit Verweis auf Tomaševski, K. (1987): The right to food: Guide through applicable international law, xviii. 
states must take whatever steps are needed to ensure its realization and international cooperation is mandatory and not subject to consent. ${ }^{31}$

Diese Definition des gewohnheitsrechtlichen Gehalts des Rechts auf Nahrung ist zu präzisieren.

I. Recht, frei von Hunger zu leben

\section{Relativer Status}

Die Gewährleistung des Rechts, frei von Hunger zu leben, kann nach dem derzeitigen Stand kaum als absolute Pflicht angesehen werden. Selbst der UN-Sozialausschuss unterscheidet zwischen der objektiven Unfähigkeit und der mangelnden Bereitschaft einer Vertragspartei, die Befriedigung des zur Vermeidung von Hunger unverzichtbaren Mindestbedarfs an Nahrung sicherzustellen. ${ }^{32}$ Nur wenn einem Staat Mittel zur Verfügung stehen und er diese wissentlich ungenutzt lässt, liegt ein Paktverstoß vor. ${ }^{33}$

Auch die mit Blick auf das Recht auf Fürsorge angeführten Urteile sprechen nicht einheitlich für eine absolute Garantie des Rechts auf Hungerfreiheit. Nach der Rechtsprechung des Interamerikanischen Menschenrechtsgerichtshofs begründen Hungerzustände an sich keine Menschenrechtsverletzung. Der Gerichtshof will den Vertragsstaaten keine unangemessenen Bürden auferlegen und stellt darauf $a b$, ob die Regierung im Rahmen der verfügbaren Ressourcen zeitnah und mit der notwendigen Priorität Maßnahmen zur Gewährleistung eines würdigen Lebens eingeleitet hat. ${ }^{34}$

31 Narula 2006 (Fn. 1), S. 81.

32 CESCR, General Comment 12 - the right to food, Rn. 17.

33 Ebd. Oben $\S 2$ C 5 d (S. 72ff.).

34 IAGMR, Sawhoyamaxa (oben $\S 5$ Fn. 31): "In order for this positive obligation to arise, it must be determined that at the moment of the occurrence of the events, the authorities knew or should have known about the existence of a situation posing an immediate and certain risk to the life of an individual or of a group of individuals, and that the necessary measures were not adopted within the scope of their authority which could be reasonably expected to prevent or avoid such risk" (Rn. 155). 


\section{Gefangene}

Eine ausreichend gefestigt Staatenpraxis und Rechtsüberzeugung von einem absoluten Recht besteht jedenfalls mit Blick auf Personen, die in einer staatlichen Haftanstalt untergebracht sind. Das Gebot zur angemessenen Ernährung von Häftlingen ist von unterschiedlichen Gerichten und Menschenrechtsorganen insbesondere aus dem Recht auf Leben abgeleitet worden. ${ }^{35}$

Die Pflicht zur ausreichenden Verpflegung gehört zu den Mindestgrundsätzen für die Behandlung der Gefangenen, die durch den UN-Wirtschafts- und Sozialausschuss bekräftigt wurden. ${ }^{36}$ Das Komitee für die Überwachung der Anti-Folter-Konvention ${ }^{37}$ vertritt die Ansicht, dass der Nahrungsentzug für Gefangene einer verbotenen unmenschlichen und erniedrigenden Behandlung gleichzustellen ist. ${ }^{38}$ Das Internationale Komitee des Roten Kreuzes zählt das Gebot, Gefangene mit angemessener Nahrung, Wasser, Unterkunft und Medizin zu versorgen, zu den gewohnheitsrechtlich geltenden Regeln des humanitären Völkerrechts. ${ }^{39}$ Damit enthält das Konfliktvölkerrecht ein zwingendes Gebot, das alle Staaten zugleich in Friedenszeiten zu beachten haben.

\section{Recht auf angemessene Ernährung}

Zudem kann bestimmten Geboten und Verboten, die der UN-Sozialausschuss aus dem Recht auf angemessene Ernährung (Art. 11 I IPwskR) ab-

35 Oben $\S 5$ A IV (S. 188ff.).

36 Standard Minimum Rules for the Treatment of Prisoners, Genf 1955, ECOSOC, Resolution 663 C (XXIV) v. 31.07.1957 u. Resolution 2076 (LXII) v. 13.05.1977.

37 Konvention gegen Folter und andere grausame, unmenschliche oder erniedrigende Behandlung oder Strafe, v. 10.12.1984, U.N.T.S. Band. 1465, S. 85.

38 Committee against Torture, Concluding Observations - Argentina, v. 10.10.2004, CAT/C/CR/33/1, Rn. 6h.

39 ICRC, Customary IHL Database, rule 118: "Persons deprived of their liberty must be provided with adequate food, water, clothing, shelter and medical attention", abrufbar unter: <www.icrc.org/customary-ihl/eng/docs/vl_rul_rule118> (Stand Mai 2014). 
leitet, im Lichte der aufgezeigten Staatenpraxis, ein gewohnheitsrechtlicher Status zugesprochen werden. ${ }^{40}$

\section{Formalpflichten}

Staaten sind gewohnheitsrechtlich verpflichtet, nationale Strategien und Programme zur progressiven Verwirklichung des Rechts auf Nahrung zu entwickeln. Diese Formalpflichten, die der UN-Sozialausschuss in der Allgemeinen Bemerkung Nr. 12 hervorgehoben hat, ${ }^{41}$ sind in den FAOLeitlinien zum Recht auf Nahrung (2004) konkretisiert ${ }^{42}$ und werden in sämtlichen Resolutionen der UN-Generalversammlung zum Recht auf Nahrung bekräftigt, ${ }^{43}$ die alle Staaten dazu ermutigen

to take steps with a view to progressively achieving the full realization of the right to food, including steps to promote the conditions for everyone to be free from hunger and, as soon as possible, to enjoy fully the right to food and to create and adopt national plans to combat hunger. ${ }^{44}$

\section{Spezifische Unterstützungs- und Förderprogramme}

Zudem sind alle Staaten verpflichtet, spezifische Unterstützungs- und Förderprogramme für Nahrungssicherheit zu implementieren. Diese müssen angesichts der umfassenden Ratifizierung der Frauen- und Kinderrechtskonvention sowie des Abkommens über die Rechte von Menschen mit Behinderungen auf die besonderen Bedürfnisse dieser Bevölkerungsgruppen ausgerichtet werden. ${ }^{45}$ Die ,right to food“-Resolutionen heben hervor, dass Programme insbesondere für Menschen mit Behinderung zugänglich

$40 \mathrm{Zu}$ unpräzise ist die Position, die sämtlichen aus dem IPwskR ableitbaren Pflichten sogleich einen gewohnheitsrechtlichen Status zuspricht, so aber Niada 2006 (Fn. 1), S. 173.

41 CESCR, General Comment 12 - right to food, Rn. 21 bis 28.

42 FAO-Leitlinien 2004 (oben $\S 3$ Fn. 142), Leitlinie Nr 3.1.

43 Zuletzt UNGA, Resolution v. 18.12.2014, RES 69/177, Rn. 40.

44 Ebd., Rn. 11.

45 Oben $\S 1$ A II 2 bis 4 (S. 35ff.). 
$\operatorname{sein}^{46}$ und Müttern sowie Kindern (insbesondere Schwangeren und Kindern unter zwei Jahren) zu Gute kommen müssen. ${ }^{47}$

a. Kinder

Der Ausschuss für die Rechte von Kindern hat die Mangelernährung von Kindern neben vermeidbaren Krankheiten als das größte Hindernis für die Verwirklichung des Rechts auf Leben in der frühen Kindheit bezeichnet. ${ }^{48}$ Die Vertragsstaaten sollen das Überleben und die Entwicklung des Kindes in umfassender Weise durch die Verwirklichung aller Konventionsbestimmungen sicherstellen und insbesondere das Recht auf Gesundheit und angemessene Ernährung garantieren. ${ }^{49}$ In seiner Kommentierung des Rechts auf Gesundheit (2013) fordert der Ausschuss die Bereitstellung angemessener und nahrhafter Nahrung sowie die Vergabe von Mahlzeiten in Schulen..$^{50}$

b. Schwangere und stillende Mütter

Eine absolute Einstandspflicht besteht gegenüber schwangeren Frauen und stillenden Müttern (Art. 12 II Frauenrechtskonvention). Dieses Übereinkommen enthält im Gegensatz zu anderen besonderen Menschenrechtsverträgen keinen Ressourcenvorbehalt. ${ }^{51}$ Der zuständige Ausschuss für die Beseitigung der Diskriminierung der Frau hat die Vertragsstaaten aufgefordert, darzulegen, in welchem Umfang sie kostenlose Dienste zur Gewährleistung einer sicheren Schwangerschaft, Geburt und frühen Mutterschaft bereitstellen. ${ }^{52}$

46 Zuletzt UNGA Resolution v. 18.12.2014, RES 69/177, Rn. 9.

47 Ebd., Rn. 10.

48 CRC, General Comment No. 7 (2005) - Implementing child rights in early childhood, CRC/C/GC/7/Rev. 1, v. 20.09.2006, Rn. 10.

49 Ebd., Rn. 27.

50 CRC, General Comment No. 15 (2013), v. 17.04.2013, CRC/C/GC/15, Rn. 43ff.

51 Vgl. Ressourcenvorbehalt in Art. 4 Satz 2 der Kinderrechtskonvention und Art. 4 II der Behindertenrechtskonvention.

52 CEDAW, General recommendation No. 24 - twentieth session, 1999 article 12 women and health, Rn. 27. 
Dieses Gebot bindet auch die fünf Staaten, welche die Frauenrechtskonvention nicht ratifiziert haben. ${ }^{53}$ Denn eine Pflicht zur Versorgung hilfsbedürftiger schwangerer Frauen und stillender Mütter ergibt sich zugleich aus dem Recht auf Leben (Art. 6 IPbpR), das ebenfalls Maßnahmen zur Verringerung der Kindersterblichkeit erfordert. ${ }^{54}$ Der Ausschuss für die Rechte des Kindes hat zugleich in überzeugender Weise eine Pflicht zur Unterstützung schwangerer Frauen und stillender Mütter, deren angemessene Ernährung unverzichtbar ist, um die gesunde Entwicklung von Embryos und Säuglingen zu gewährleisten, aus der UN-Kinderrechtskonvention abgeleitet. ${ }^{55}$

\section{Schutzdimension}

\section{a. Recht auf Selbstversorgung}

Die völkerrechtliche Entwicklung spricht schließlich für die Annahme, dass das Recht auf Selbstversorgung jedenfalls als „status negativus“ gewohnheitsrechtlich gilt. Dieser legt dem Staat die Pflicht auf, Kleinproduzierende, die sich selbst versorgen, vor Umweltschäden und willkürlichen Vertreibungen zu bewahren. Das Verbot Menschen, die sich selbst versorgen, die Subsistenzgrundlage zu entziehen, findet seinen Ausdruck ebenfalls in einer gewohnheitsrechtlichen Regel des humanitären Völkerrechts: Danach ist es untersagt, Nahrungsmittel, zur Erzeugung von Nahrungsmitteln genutzte landwirtschaftliche Gebiete, Ernte- und Viehbestände sowie sonstige Objekte zu zerstören, die für die Zivilbevölkerung lebensnotwen$\operatorname{dig}$ sind..$^{56}$

53 Iran, Palau, Somalia, Sudan und die Vereinigten Staaten von Amerika haben die Konvention nicht ratifiziert, vgl. U.N.T.C., Chapter IV, No. 8 (Stand Mai 2015).

54 CCPR, General Comment 6 - right to life, Rn. 5.

55 CRC, General Comment No. 15, Rn. $43 \mathrm{ff}$.

56 ICRC, Customary IHL Database, Rule 54: Attacks against Objects Indispensable to the Survival of the Civilian Population, <www.icrc.org/customary-ihl/eng/docs/ vl_rul_rule54> (Stand Mai 2014). 


\section{b. Indigene Rechte}

Wenngleich das ILO-Abkommen Nr.169, das indigenen Völkern ein Recht auf Land und Territorium garantiert, bislang nur von 22 Staaten ratifiziert wurde, ${ }^{57}$ repräsentiert dieses einen völkergewohnheitsrechtlichen Standard. Wie der Interamerikanische Menschenrechtsgerichtshof im Fall „Kischwa“ (2012) nachgezeichnet hat, haben zahlreiche Mitgliedstaaten der Organisation Amerikanischer Staaten die ILO-Regeln zur vorherigen Konsultation durch verfassungsrechtliche oder gesetzliche Bestimmungen inkorporiert, die zudem in vielen Ländern durch höchstrichterliche Rechtsprechungen getragen werden. ${ }^{58}$

Ein länderübergreifender Konsens über die Schutzwürdigkeit indigener Landrechte ergibt sich aus der UN-Erklärung über die Rechte indigener Völker, die im September 2007 von der Generalversammlung der Vereinten Nationen verabschiedet wurde. ${ }^{59}$ Auch die Leitlinien über Nutzungsrechte des FAO-Welternährungskomitees (2012) sehen Staaten im Fall eines Ressourcenprojekts, das die Landrechte indigener Völker beeinträchtigen könnte, in der Pflicht, eine freie vorherige und informierte Zustimmung des Volkes einzuholen. ${ }^{60}$

\section{Zwingende Regel des Völkerrechts}

Nach Anthony Paul Kearns hat das Recht auf Nahrung zugleich den Status einer zwingenden Regel des Völkerrechts (ius cogens) erreicht. ${ }^{61}$ Nach Art. 53 Satz 2 der Wiener Vertragsrechtskonvention ist unter dem Begriff des zwingenden Rechts eine Regel zu verstehen, die von der internationalen Staatengemeinschaft in ihrer Gesamtheit angenommen und als eine Norm anerkannt wird, von der nicht abgewichen werden darf. Eine ,ius

57 Nur ein afrikanisches Land (Zentralafrikanische Republik) und ein asiatischer Staat (Nepal) sind bislang Vertragsparteien geworden. Siehe Liste der Ratifikationen, Normlex, Information System on International Labour Standards, C169 (Stand Juli 2014).

58 IAGMR, Kischwa (oben $\S 5$ Fn. 112), Rn. 164.

59 UNGA, Resolution v. 13.09.2007, UN Declaration on the Rights of Indigenous, RES 61/295.

60 Vgl. CFS-Leitlinien 2012 (oben $\S 4$ Fn. 84), Nr. 9.9.

61 Kearns 1998 (Fn. 1), S. 255; ablehnend Narula 2006 (Fn. 1), Rn. 428 (S. 67). 
cogens"-Norm kann nur durch eine andere zwingende Regel aufgehoben werden und bewirkt die Nichtigkeit entgegenstehenden Rechts. ${ }^{62}$

\section{Rechtspraxis}

In der gerichtlichen Praxis wurde Menschenrechten mehrfach ein „,ius cogens“-Status zugeschrieben: Im bereits erwähnten „Domingues“-Fall hat die Interamerikanische Menschenrechtskommission das Verbot der Todesstrafe von Jugendlichen unter 18 Jahren als eine zwingende Regel gewertet, an welche die Vereinigten Staaten von Amerika (ungeachtet ihres Vorbehaltes gegenüber Art. 6 V IPbpR) gebunden seien. ${ }^{63}$ Das Jugoslawientribunal hat in seiner „Furundžija“-Entscheidung (1998) das Folterverbot als ,,ius cogens"-Norm eingestuft und damit die Nichtigkeit eines nationalen Amnestiegesetzes begründet. ${ }^{64}$ Im Fall „Kadi““ (2005) argumentierte das Europäische Gericht erster Instanz, dass die Europäische Union nicht an Resolutionen des UN-Sicherheitsrates gebunden sei, soweit diese gegen zwingende Menschenrechte verstoßen. ${ }^{65}$ Die aufgezeigten Fälle zeigen, dass das Konzept zwingender Menschenrechte als Hierarchiesierungsformel genutzt werden kann, um Normkonflikte zu entscheiden.

\section{Das Recht auf ein Leben ohne Hunger}

In der Literatur wird neben dem Gewalt- und Interventionsverbot elementaren Menschenrechten (wie dem Folterverbot) ein zwingender Charakter zugesprochen. ${ }^{66}$ Dazu ist zumindest das Recht auf ein Leben ohne Hunger (Art. 11 II IPwskR) zu zählen. In diesem Sinne geht auch Stefan Kadel-

62 Kadelbach, S. (1992): Zwingendes Völkerrecht, S. 324ff.

63 IAMRK - Domingues (Fn. 16).

64 ICTY, Prosecutor v. A. Furundzija, Trail Chamber, Urt. v. 10.12.1998, IT-95-17/1, ILM 38 (1999), S. 349 ff., Rn. 155.

65 Im konkreten Fall kritisierte das EuG den fehlenden Rechtsschutz gegen das Einfrieren der Konten von Terrorverdächtigen durch den Sicherheitsrat, verneinte jedoch eines ,ius cogens“-Verstoß, EuG, Urt. v. 21.09.2005, Rs. T-315/01, 2005, Kadi u.a. und T-306/01, Yusuf u.a.

66 Siehe Hannikainen, L. (1988): Peremtory Norms (jus cogens) in International Law, S. 425ff. 
bach davon aus, dass der zwingende Charakter des Rechts, frei von Hunger zu leben, Staaten verbietet, internationale Hilfsaktionen zur Linderung akuter Hungerkrisen zu unterbinden. ${ }^{67}$

Solche Kollisionsfälle hat Kearns nicht vor Augen, der den zwingenden Status des Rechts auf Nahrung als Argument für die besondere Dringlichkeit von effektiven Anti-Hunger-Maßnahmen anführt: Demnach sei jeder Staat gezwungen, alle notwendigen Maßnahmen zur Sicherung einer angemessenen Ernährung seiner Bevölkerung zu ergreifen. ${ }^{68}$ Aufgrund des „ius cogens"-Status des Rechts auf ein Leben ohne Hunger ist jede Norm, die sich in offensichtlichem Widerspruch zu diesem fundamentalen Recht setzt, als nichtig anzusehen. Eine völkervertragliche Bestimmung, die Personen oder Gruppen den Zugang zu Nahrung verweigert und diese dadurch Unter- oder Mangelernährung preisgibt, wäre unwirksam. Neben solchen schwer vorstellbaren unmittelbaren Diskriminierungen wäre z.B. eine Vereinbarung als nichtig anzusehen, die Kleinproduzierenden oder indigenen Völkern den Zugang zu natürlichen Ressourcen entzieht, die sie zur Eigenversorgung benötigen, soweit keine vorherige Konsultation und angemessen Entschädigung vorgesehen sind.

\section{Einordnung}

Das Recht auf Nahrung ist Bestandteil des universellen Völkergewohnheitsrechts. Jeder Staat hat die Pflicht Nahrungssicherheitsstrategien einzuführen sowie Unterstützungsprogramme für Menschen mit Behinderungen, Frauen und Kindern zu implementieren. Gegenüber Häftlingen, Schwangeren und Stillenden besteht eine absolute Einstandspflicht, die nicht unter den Ressourcenvorbehalt fällt. Zudem kommt der negativen Dimension des Rechts auf Nahrung, die Kleinproduzierende und indigene Gemeinschaften vor willkürlichen Vertreibungen und Zerstörung ihrer Lebensgrundlage schützt, eine gewohnheitsrechtliche Geltung zu. Schließlich ist das Recht auf ein Leben ohne Hunger (Art. 11 II IPwskR) zum zwingenden Völkerrecht (ius cogens) zu zählen, von dem keine Rechtsnorm abweichen darf.

67 Kadelbach 1992 (Fn. 62), S. 289.

68 Kearns 1998 (Fn. 1), S. 255. 
Die Einstufung des Rechts auf Nahrung als gewohnheitsrechtliche Norm ist im Hinblick auf die im nächsten Kapitel zu behandelnde globale Dimension der Hungerkrise von Bedeutung. Aus ihr folgt, dass alle Völkerrechtssubjekte, die Nicht-Vertragsparteien des UN-Sozialpakts und Internationale Organisationen eingeschlossen, an das ,right to food“ gebunden sind. 